\title{
Photofrin-mediated photodynamic therapy for treatment of early stage laryngeal malignancies
}

\author{
Vanessa Gayl Schweitzer • Melissa L. Somers
}

Received: 2 April 2010/Accepted: 22 July 2010/Published online: 10 August 2010

(C) Springer-Verlag 2010

\begin{abstract}
To evaluate the efficacy of PHOTOFRINmediated photodynamic therapy (PDT) for the treatment of Tis-T1N0M0 squamous cell carcinoma ( $\mathrm{SqCCa})$ of the larynx in patients not amenable to or who failed conventional head and neck treatment. This is a retrospective study of 26 patients with early stage Tis-T1 SqCCa of the larynx treated with PHOTOFRIN-mediated PDT. Intravenous PHOTOFRIN (porfimer-sodium) (dose $2.0 \mathrm{mg} / \mathrm{kg}$ ) was administered outpatient, followed by intraoperative photoactivation at $630 \mathrm{~nm}$ via fiberoptic microlens surface

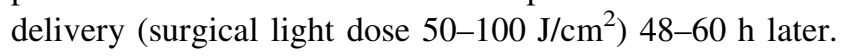
As much as 16 out of 26 patients (62\%) have demonstrated complete remission (average follow-up 40 months). There were 10 patients who were noted to have partial remission with recurrence observed 2-33 months subsequently retreated with either repeated PDT therapy or conventional therapy. PHOTOFRIN-mediated photodynamic therapy can be used as a primary modality to treat Tis-T1N0M0 tumors of the larynx or for treatment for those who have failed prior surgery and/or radiation therapy. PDT allows for preservation of function and structure to maintain or improve voice with absence of systemic toxicity. Patients may have multiple drug administrations and laser light retreatment for local disease control.
\end{abstract}

Keywords Photodynamic therapy $\cdot$ Larynx $\cdot$ Photofrin · Head and neck cancer

This paper was presented at the Middle Section Meeting in Orlando, FL on $2 / 4 / 2010$.

V. G. Schweitzer $(\bowtie) \cdot$ M. L. Somers

Department of Otolaryngology/Head and Neck Surgery,

Henry Ford Medical Group, 2799 W Grand Blvd.

(K8 Clinic Building), Detroit, MI 48202, USA

e-mail: vschwei1@hfhs.org

\section{Introduction}

It is estimated that in the United States there will be diagnosed 12,290 new cases of laryngeal cancer in 2009 [1]. Potential etiologies include tobacco, marijuana, and alcohol abuse, as well as tumorigenesis via genetic predisposition or epigenetic pathways involving DNA and histomodifications resulting in the heritable silencing of genes without a change in their coding sequence and/or aberrant DNA methylation whereby methyl groups are bound to the promoter sequence of tumor suppressor genes causing inactivation of the gene [2].

Numerous clinical investigations have demonstrated the effectiveness of photodynamic therapy (PDT) with dihematoporphyrin ether (PHOTOFRIN), 5-aminolevulinic acid (ALA), and meta-tetrahydroxyphenylchlorin (mTHPC) (FOSCAN) in the treatment of minimally invasive early squamous cell carcinoma (SqCCa) of the head and neck due to the ability of the activating 630-664 $\mathrm{nm}$ laser light to penetrate $0.5-1 \mathrm{~cm}$ into tissues resulting in destruction of microscopic tumor with preservation of protected normal tissues [3-8].

The most extensively studied first generation photosensitizer is PHOTOFRIN which is photoactivated at $630 \mathrm{~nm}$, penetrating tissues to a depth of $0.5-1.0 \mathrm{~cm}$, but limits curative treatment of deeper invasive solid tumors. Therefore, PHOTOFRIN-mediated PDT with a surface tissue effect of $1.0 \mathrm{~cm}$ is very effective for treatment of superficial, microinvasive squamous cell, basal cell, and adenocarcinomas with surface illumination using microlens (ML) fiberoptic tips. Treatment of deep seated, intraluminal bulky, or intracavitary tumors requires endoscopic placement of cylindrical diffusers (CDs), intracavitary placement of spherical diffusers or CDs, or interstitial placement of CDs for bulky solid tumors. 
Currently, photodynamic therapy has been approved for clinical oncologic treatment in the United States, European Union, Canada, Russia, and Japan. In the United States US Food and Drug Administration approval has been given for PHOTOFRIN-mediated photodynamic therapy in obstructing esophageal and endobronchial tumors as well as minimally invasive endobronchial non-small cell carcinoma, and Barrett's esophagitis with high grade dysplasia and carcinoma in situ [9]. PHOTOFRIN-mediated photodynamic therapy is used off label in treatment of unresectable malignant brain tumors, prostate cancer, cholangiocarcinoma, and head and neck cancer. The senior author (VS) has also used PHOTOFRIN-mediated photodynamic therapy for treatment of adult and juvenile laryngeal papillomatosis, Tis-T2NOM0 laryngeal carcinoma, AIDS-related mucocutaneous Kaposi's sarcoma, aggressive squamous cell carcinoma and basal cell skin malignancies, and palliative treatment of end stage head and neck cancer [4].

In the European Union, PDT has been approved for treatment of both early stage head and neck cancers and palliative treatment of head and neck cancer using the photosensitizer meta-tetrahydroxyphenylchlorin (FOSCAN) and for treatment of non-melanoma skin cancers using the photosensitizer Metvix [5, 10, 11]. Data are now available for over 1,500 patients treated with PDT using PHOTOFRIN, 5-aminolevulinic acid (ALA), and metatetrahydroxyphenylchlorin (FOSCAN) for the treatment of head and neck cancers. Multi-Institutional Phase II-III clinical trials in Europe evaluating FOSCAN-PDT treatment for head and neck cancers have demonstrated efficacy for this minimally invasive therapy for treatment of primary and recurring cancers as well as palliative treatment of refractory head and neck cancers using FOSCAN photodynamic therapy [7]. The USA FDA rejected FOSCAN for treatment of oral cavity carcinoma because of extensive normal mucosal damage (Dougherty T, PhD, Roswell Park Institute, Buffalo, NY, personal communication).

The purpose of this report is to summarize encouraging clinical data in 26 early stage laryngeal cancer patients treated at Henry Ford Health System by the senior author from 1986 to 2009. Summarized are details of the light dosimetry, treatment technique, procedure precautions, and possibilities for future applications of PDT in malignant head and neck early stage disease corroborating studies by multiple other authors.

\section{Materials and methods}

Clinical use of porfimer-sodium (PHOTOFRIN) for head and neck cancer in the United States is still investigational and follows specific FDA approved guidelines. It was previously administered by Quadra Logic Technologics, Inc., Vancouver, British Columbia, Canada and currently is distributed by Axcan Pharma Inc., Birmingham, Alabama. The study was performed following human rights approval through the Henry Ford Health System IRB.

Patients were selected if they had (1) moderate to severe dysplasia or squamous Tis of the larynx demonstrated on biopsy; (2) stage I (T1N0M0) squamous cell carcinoma of the larynx demonstrated on biopsy; (3) either already received or did not desire alternate therapy, such as cold knife surgery, radiation therapy, or $\mathrm{CO}_{2}$ laser therapy; or (4) recurrent lesions still staged as Tis or T1.

Patients were excluded if they (1) were pregnant; (2) had hypersensitivity to porphyrins; (3) had impaired hepatic or renal function; or (4) had stage II or more advanced stage disease.

PHOTOFRIN was supplied in $2.5 \mathrm{mg} / \mathrm{ml}$ and $30 \mathrm{ml}$ vials, stored lyophilized at $0^{\circ} \mathrm{C}$ in the dark until used, when reconstituted with D50 at room temperature immediately before administration. It was administered intravenously over $10 \mathrm{~min}$ at a dose of $2 \mathrm{mg} / \mathrm{kg}$ in the outpatient setting. As much as $48-60 \mathrm{~h}$ following administration, patients were treated in outpatient surgery and were placed under general anesthesia via Hunsaker-Mon Jet ventilation with an $\mathrm{FiO}_{2}$ of $100 \%$. Photoactivation was administered from an Argon-pumped Rhodamine B-dye Coherent $(630 \mathrm{~nm})$ laser, a Laserscope Series 600 PDT dye module $(630 \mathrm{~nm})$, or a Diomed portable laser $(630 \mathrm{~nm})$. Light was delivered to the tumor bed through a $400 \mu \mathrm{m}$ fused silica fiber using a flat ML tip with dosimetry guidelines $50-100 \mathrm{~J} / \mathrm{cm}^{2}$ (Fig. 1). There are no previous large institutional studies regarding optimal light dose and dosages were based on the principal investigator's prior experience and other investigators $[4,7]$. If a patient required repeated administration of PDT, light dosimetry of $80-100 \mathrm{~J} / \mathrm{cm}^{2}$ was utilized.

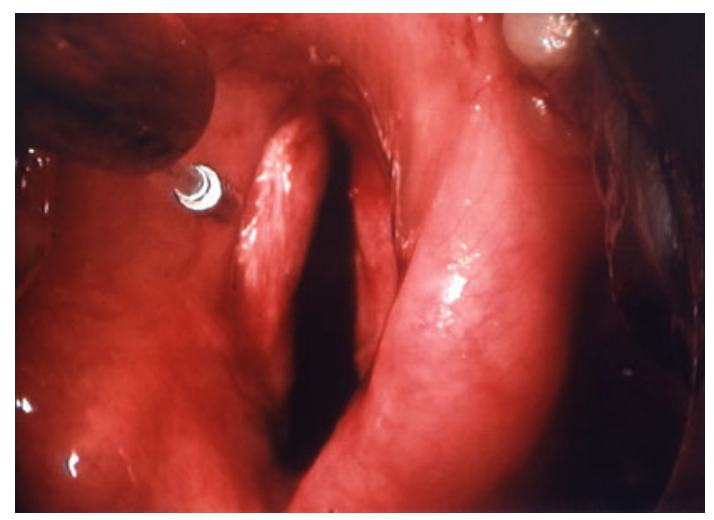

Fig. 1 Intraoperative positioning of microlens fiber using HunsakerMon Jet ventilation. Patient with Tis-T1 laryngeal cancer with history of prior $\mathrm{CO}_{2}$ laser treatment 
Hospital based, drug-related photosensitivity reactions were minimal due to education of hospital support staff including residents, housekeeping, ambulatory surgery operating room, and inpatient nursing staff. Patients signed and detailed informed consents outlining drug-related information and reviewed pharmaceutical brochures and videotapes regarding photosensitivity precautions, including clothing and travel-related measures to minimize post-operative photosensitivity within the first 4 weeks following drug injection. During daylight hours, patients are instructed to wear long sleeves shirts, slacks, gloves, socks, shoes, and a wide brimmed hat when outdoors. Tightly woven light colored fabrics are preferred. Patients are instructed to wear dark sunglasses (less than $4 \%$ white light transmittance). They are instructed to wear protective clothing and sunglasses even on cloudy days and particularly while driving a car. Indoor lighting is encouraged in order to facilitate photobleaching of the drug from the skin. Patients are to avoid skylights, direct sunlight, undraped windows, but other activities, such as computer work, watching television, movie theaters, etc. are encouraged. Intraoperatively all finger jewelry is removed, finger pulse oximeters (wave length $600 \mathrm{~nm}$ ) are rotated every $15 \mathrm{~min}$, and no direct overhead surgical lights unless covered with yellow acrylic filters passing $600 \mathrm{~nm}$ wavelength are used. All skin and mucosal surfaces not in the treatment field are completely covered. Eye and dental protection secured, and headlights worn for intraoperative procedures.

Perioperative care consisted of intraoperative steroids (10 mg intravenous decadron after treatment), occasional medrol dose pack postoperatively, and analgesics for 1-2 weeks (which may include morphine elixir, Tylenol with codeine elixir).

Patients were examined for pain, bleeding, glottic swelling, inflammation, and tumor slough postoperatively and were monitored for recurrence (Figs. 2, 3). Treatment response for laryngeal disease in this series following physical evaluation is defined as: (1) complete response (CR) - no visual or biopsy proven disease up to 2 years or length of follow-up, (2) partial response (PR)-reduction of $50 \%$ or more in maximum diameter of affected area or $50 \%$ reduction in number of visible tumors or negative visual exam with positive random biopsy of treatment site, or (3) no response (NR) - reduction of diameter of initially affected area by less than $50 \%$, increase in the size of the tumor and/or new tumor growth (progression of disease). Biopsies are performed within 6-8 weeks of PDT treatment for any persistent lesions and periodically to verify response as per the decision by the senior author to document recurrence or until patient was lost to follow up.

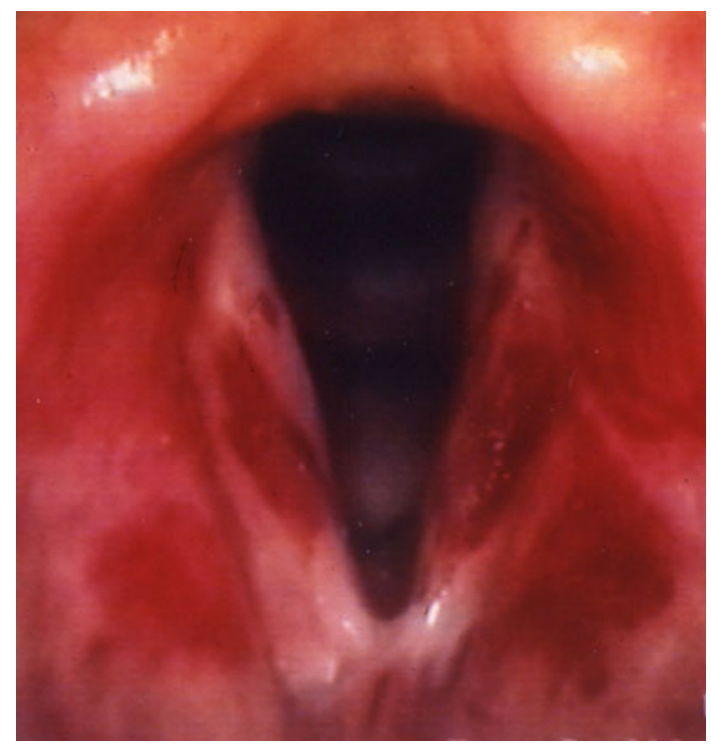

Fig. 2 Post-operative day 18 following PDT therapy. Evaluation of the glottis using videostroboscopy demonstrates typical petechial changes

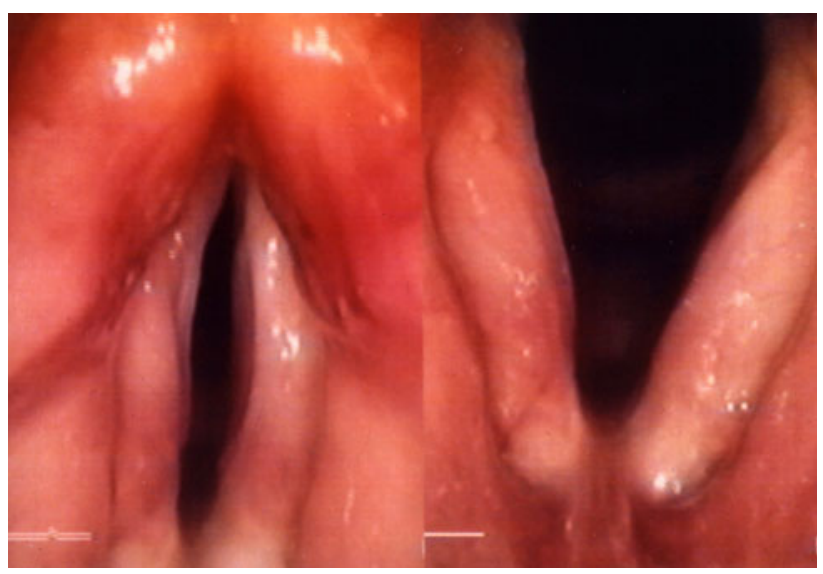

Fig. 3 Post-operative evaluation of the glottis 2 years following therapy. Patient does not have evidence of disease 14 years post treatment

\section{Results}

There were a total of 26 patients who were treated and monitored from 1985 through June 2008. Of the 26 patients, 5 were female and 21 were male. The ages ranged between 29 and 85 years at first treatment. Three patients had undergone radiation therapy prior to PDT and 2 patients had undergone $\mathrm{CO}_{2}$ laser excision prior to PDT therapy. The remaining 21 had primary disease. As much as 8 patients had evidence of T1 disease and 18 patients had carcinoma in situ. The patients were followed up for an average of 40 months (range 2 weeks to 156 months).

A total of 16 out of 26 manifested complete local remission (62\%). One patient (patient 8) died 2 weeks 


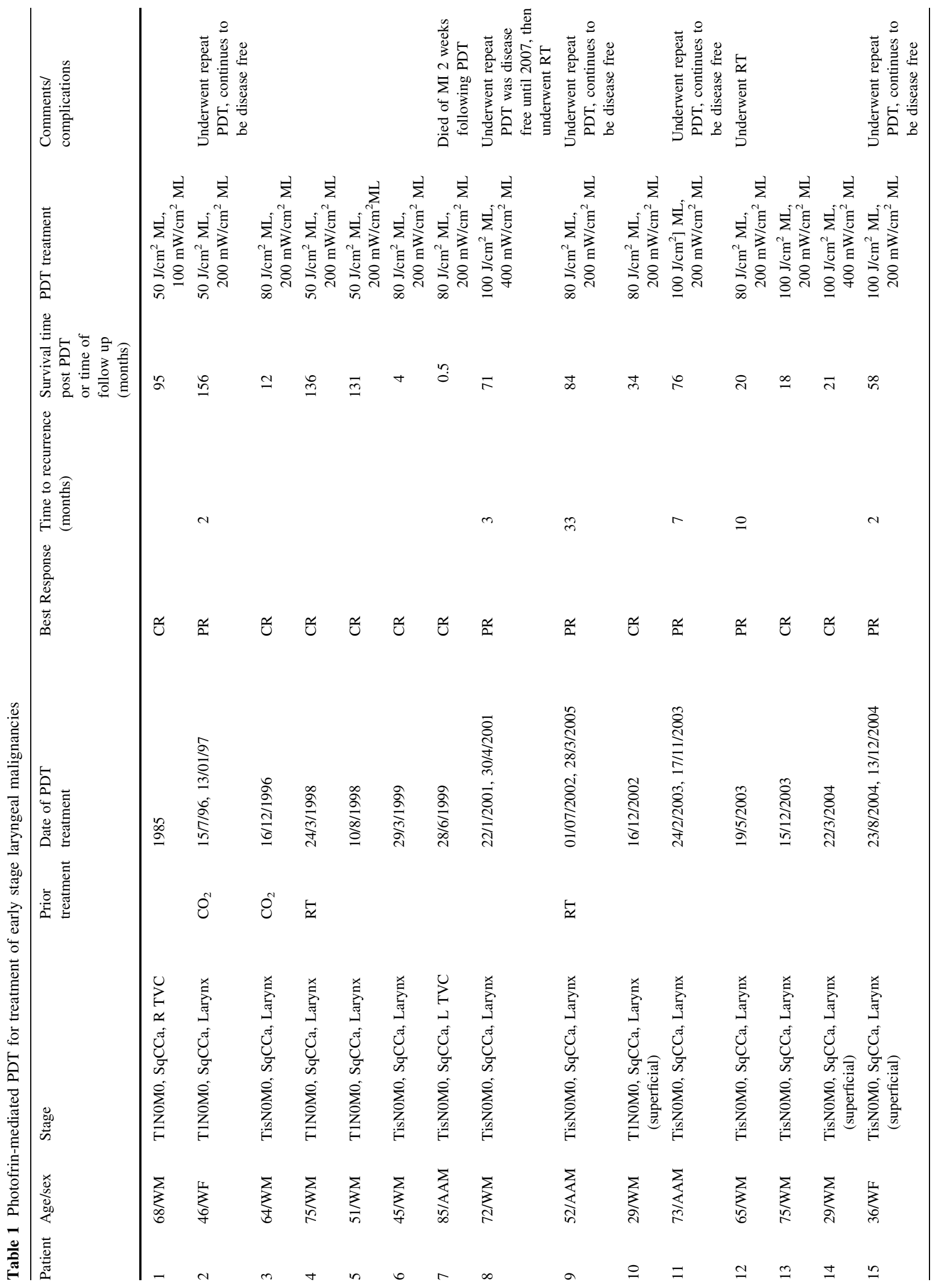




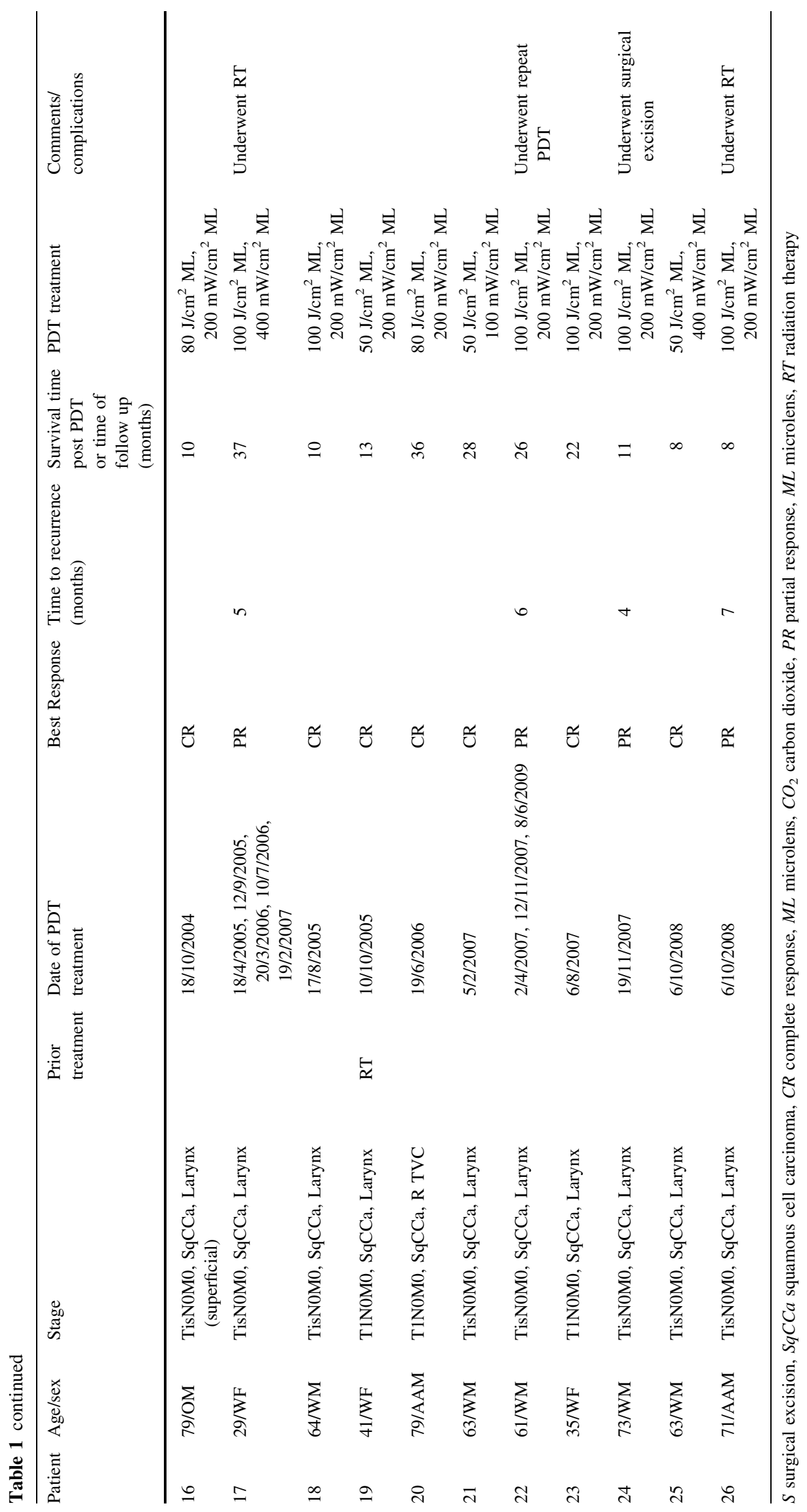


following therapy from a myocardial infarction not related to the PDT treatment. Details of this patient series, treatment responses, and time of follow up are listed in Table 1. The average length of follow up for this group was 36 months (range 2 weeks to 136 months).

There were 10 patients who were noted to have partial remission with recurrences observed between 2 and 33 months subsequently retreated with either repeated PDT therapy or conventional therapy. One of the partial remission patients was noted to originally have a T1 lesion and had previously undergone $\mathrm{CO}_{2}$ laser surgery prior to the PDT therapy. The other 9 patients were noted to be Tis at original presentation.

As much as 7 out of the 10 patients underwent repeated PDT therapy. Two patients required more than 2 treatments with PDT. Of the 2 patients who required more than 2 treatments, one subsequently had radiation therapy for cure following 5 PDT treatments, and one continues to be disease free after a third PDT treatment. One patient developed a new primary 6 years after the second PDT treatment and then underwent radiation therapy for cure. The total complete response rate for the patients who received one or more PDT treatments with 2 years of subsequent disease free follow-up is $84 \%$. A total of 3 out of the 10 patients who developed recurrence and did not elect to undergo repeated PDT underwent radiation therapy for cure and one patient underwent surgical excision for cure.

Furthermore, for all patients voice quality via perceptual analysis was stable or improved post treatment.

\section{Discussion}

Many patients with early stage Tis-T1 laryngeal squamous cell carcinoma will undergo surgical excision (partial cordectomy, cordectomy), $\mathrm{CO}_{2}$ laser therapy, radiation therapy, or a combination for persistent disease. Each of the conventional treatment modalities poses unwanted side effects with surgical excision causing structural defects leading to dysphonia and dysphagia, and ionizing radiation therapy producing side effects, such as dysphonia, hyperpigmentation, vocal cord scarring, and xerostomia.

There has been an increase in the use of photodynamic therapy (PDT) over the past few decades as an alternative for treating minimally invasive cancers of the head and neck. Rather than radiation which has a limited cGy dose, photodynamic therapy can be used multiple times without systemic toxicity. Once photochemically activated, Photofrin works by (1) irreversibly oxidizing cellular tissue, leading to free radicals, mitochondrial damage, and cell apoptosis and (2) through vascular endothelial damage [12]. It does not damage the collagen or elastin structures, so normal tissue can be regenerated following the procedure [3]. PDT does not predispose patients to the risk of osteoradionecrosis unlike those who undergo radiation therapy.

PDT resulted in complete remission in $62 \%$ of cases of Tis-T1N0M0 laryngeal squamous cell carcinomas, $84 \%$ complete response rate when looking at patients with one or more PDT treatments. This is comparable to studies published by Biel [7], demonstrating an $89 \%$ complete response rate while looking at 171 laryngeal squamous cell carcinoma patients with a 16-year follow up. A more recent prospective trial demonstrated 5 out of 6 patients $(83 \%)$ demonstrating a complete response in Tis and T1 laryngeal squamous cell carcinoma patients [8].

A minor inconvenience of PDT is that patients are photosensitive for a minimum of 4 weeks following the procedure. This can be a disadvantage to the active individual who spends a majority of their time outdoors at work or on vacation. Patients must receive extensive education regarding sun protection to prevent severe sunburns, which has never been a major problem in our series. Patients also experience hoarseness temporarily, but have been shown to subjectively have improvement in their voices overall [7].

Promising advancements that can be made in the field of PDT include newer photosensitizing agents that cause less skin photosensitivity. Newer agents can be explored that have the ability to penetrate tissues at depths greater than $1 \mathrm{~cm}$ so that more advanced tumors can also be treated with photodynamic therapy. Future studies at our institution will compare voice as observed by videostroboscopy and tumor responses following PDT therapy.

\section{Conclusion}

Photodynamic therapy is an effective first alternative to radiation therapy, $\mathrm{CO}_{2}$ laser therapy, and surgical resection for treatment of early laryngeal squamous cell carcinomas. It does not damage the underlying tissue which allows for multiple treatments, allows it to be given prior to or following other therapies. Photosensitivity is a short-term side effect. Future studies by the senior author will be focused on PDT with PHOTOFRIN as the sensitizing agent and its treatment effects on voice quality.

Conflict of interest None.

\section{References}

1. National Cancer Institute. http://www.cancer.gov/cancertopics/ types/throat

2. Spitz MR (1994) Epidemiology and risk factors for head and neck cancer. Semin Oncol 21(3):281-288 
3. Copper MP, Triesscheijn M et al (2007) Photodynamic therapy in the treatment of multiple primary tumours in the head and neck, located to the oral cavity and oropharynx. Clin Otolaryngol 32:185-189

4. Schweitzer VG (2001) PHOTFRIN-mediated photodynamic therapy for treatment of early stage oral cavity and laryngeal malignancies. Lasers Surg Med 29:305-313

5. Lorenz KJ, Maier H (2009) Photodynamic therapy with metatetrahydroxyphenylchlorin (Foscan) in the management of squamous cell carcinoma of the head and neck: experience with 35 patients. Eur Arch Otorhinolaryngol 266(12):1937-1944

6. Franco RA Jr (2007) Aminolevulinic acid $585 \mathrm{~nm}$ pulsed dye laser photodynamic treatment of laryngeal keratosis with atypia. Otolaryngol Head Neck Surg 136(6):882-887

7. Biel M (2010) Photodynamic therapy of head and neck cancers. Methods Mol Biol 635:281-293
8. Rigual NR, Thankappan K, Cooper M et al (2009) Photodynamic therapy for head and neck dysplasia and cancer. Arch Otolaryngol Head Neck Surg 135(8):784-788

9. Dougherty TJ (2002) An update on photodynamic therapy applications. J Clin Laser Med Surg 20(1):3-7

10. European Medicines Agency (2009) Science Medicines Health. (Drug name) Foscan-EPAR-product information, Annex I. Summary of product characteristics, pp 1-40. http://www.ema. europa.eu/ema/index.jsp?curl=/pages/home/Home_Page.jsp\& jsenabled=true. Accessed 2 April 2010

11. Ortiz-Pilocarpio B, Liu H (2009) Methyl aminolevulinate-PDT for actinic keratosis and superficial nonmelanoma skin cancers. Skin Ther Lett 14(6):1-3

12. Sibata $\mathrm{CH}$, Colussi VC et al (2001) Photodynamic therapy in oncology. Expert Opin Pharmacother 2(6):917-927 